\title{
Improving the Image Acquisition Rate of an Atomic Force Microscope Through Spatial Sub-sampling and Reconstruction
}

\author{
Roger A. Braker, Yufan Luo, Student Member, IEEE, Lucy Y. Pao, Fellow, IEEE \\ and Sean B. Andersson, Senior Member, IEEE, and Member, ASME
}

\begin{abstract}
Undersampling-based approaches in Atomic Force Microscopy (AFM) aim to reduce the time to acquire an image by reducing the number of measurements needed while still maintaining image quality. The approach consists of two components: data acquisition and image reconstruction. Successful practical implementation involves solving a variety of non-trivial problems. In this paper, we describe an implementation based on a collection of short scans known as $\mu$-paths, and we demonstrate it on a commercial AFM using a grating sample. Reconstructions are made from the data using a new variant of basis pursuit designed to reduce artifacts arising from the sampling pattern. The quality of the resulting images is compared to images from standard raster scans of the same regions at comparable imaging rates using both the peak signal-to-noise ratio and the structured similarity index metric and a new metric we call the relative damage index. We also compare to simple subline sampling where only a subset of the rows of the image are acquired (followed by reconstruction). These experiments show that at low sampling densities and slow scan rates, $\mu$-path scanning achieves better image quality in less time than subline sampling. Conversely, for faster scan rates subline sampling is faster and, for higher sampling densities, achieves comparable image quality.
\end{abstract}

\section{INTRODUCTION}

The Atomic Force Microscope (AFM) is a powerful instrument capable of imaging sample surface topography, material characteristics, and other surface properties, with nanometer scale precision. AFMs acquire sample information by monitoring the deflection of the cantilever caused by the tipsample interaction force. In general, feedback control is used to hold the deflection signal constant and information about the property of interest is inferred from the applied control or the measured dynamics of the cantilever [1]. Because of its versatility, spatial resolution, and ability to image in vacuum, air, and liquid, AFM is widely used in a variety of disciplines, including physics, biology, and materials science [2]-[6].

In addition to imaging static samples, AFM is used to study dynamics in systems with nanometer-scale features [3], [7]-[9]. The image acquisition time of conventional AFMs, however, is typically on the order of seconds to minutes,

Braker and Pao are with the Electrical, Computer, \& Energy Engineering Dept. at the Univ. of Colorado, Boulder, CO 80309 USA. +1 (303) 492-2360.

Luo and Andersson are with the Division of Systems Engineering, and Andersson is also with the Dept. of Mechanical Engineering, Boston Univ., Boston, MA 02215 USA.

This work was supported in part by the US National Science Foundation (NSF Grants CMMI-1234980, CMMI-1234845, CMMI-1562031, and DBI1352729), Agilent Technologies, Inc., a Palmer Endowed Chair Professorship, and the Hanse-Wissenschaftskolleg Institute for Advanced Study. severely limiting the time-scales that can be explored. Driven by the need for faster imaging, there continue to be many active research efforts to overcome this challenge. Since an AFM is fundamentally a mechanical microscope, many of the approaches to high-speed AFM (HS-AFM) have focused on modifications to the physical components. These have included the use of small, fast cantilevers [10]-[12], the development of faster actuators [13]-[15], and the application of advanced controllers [16]-[19]. By combining different techniques, there are current generation, high-end instruments that can image at rates on the order of 1-10 frames/sec [9]. However, the fastest rates are achieved only over small scan ranges and there remain many systems of interest whose dynamics are faster even than these instruments can achieve. In addition, there is a large installed base of much slower AFMs that can benefit from alternative approaches to improving imaging rates.

A complimentary class of HS-AFM approaches modifies the sampling scheme rather than the system mechanics. Alternative scan trajectories like spirals, cycloids, and Lissajous figures have been used in place of the standard raster pattern. While these trajectories are easier for the actuators to follow, allowing the tip to be moved more quickly and leading to faster image acquisition on an otherwise unmodified instrument [20]-[24], they are ultimately limited by the vertical bandwidth of the instrument for regulating the deflection signal [25].

Non-raster scan patterns can also improve imaging rate by reducing sampling rather than increasing speed. This class includes local scanning methods that use the measurements in real-time to steer the tip to focus the scan on features of interest [26]-[29]. While these have been shown to yield an order of magnitude or better improvement in imaging rate, they are limited in the class of samples that can be imaged and do not produce a full image with all the context.

An alternative group of non-raster scanning schemes, introduced in [30], [31], seeks to produce the full image but using fewer measurements than in a full raster scan. We refer to this as undersampling. The data acquisition time is then reduced by having a shorter total scan path. Taking advantage of the redundancy in many natural signals of interest, the final surface image can be recovered from the limited number of measured pixels using a variety of reconstruction methods such as inpainting or schemes based on the theory of compressive sensing (CS) [32], [33]. In addition to reduced imaging time, undersampling schemes also reduce tip-sample interactions, thereby reducing the likelihood of tip wear or sample damage. 
One simple way to create undersampling schemes is by modifying existing full scan patterns including raster, spiral, and Lissajous scanning. For example, subline sampling is generated by randomly skipping some of the horizontal lines in raster scanning [32], [34]. For spiral and Lissajous patterns, the scan parameters can be selected to ensure the trajectory only passes through a desired fraction of the pixels in the final image. The scanning time for these smooth undersampling patterns can be estimated based on the proportion of the pixels in the trajectory. However, results in the literature of CS make clear that in general randomness in the sampling pattern is essential for creating good reconstructions.

In AFM, implementing a truly random pattern requires that the tip be engaged with the surface to collect a measurement, lifted and moved to the new location, and engaged again. Because the re-engagement process is typically slow, this can lead to excessively long image acquisition times and negate the gains from undersampling [31]. In [35], we introduced an undersampling scheme, called a $\mu$-path pattern, which consists of short randomly placed, horizontal scans (illustrated in Fig. 1). It is designed to balance randomness (to ensure good reconstruction) with continuous scanning (to reduce the number of tip engagements).

Our previous work on the $\mu$-path pattern, using theoretical calculations, simulations, and a preliminary implementation, demonstrates that the approach can reduce scanning time while maintaining faithful image reconstruction [35]-[37]. The main contributions of this paper relative to that past work are:

- We introduce a new reconstruction algorithm designed to minimize artifacts arising from the structure of the $\mu$ path pattern. The algorithm is described and compared in simulation to existing reconstruction methods in Sec. II.

- In our prior experimental prototype [37], we used integral controllers for all three axes for both raster and $\mu$-path scans. Here, we design compensators for each axis using standard loop-shaping techniques (Sec. IV-C) that result in a substantial increase in closed-loop bandwidth (e.g., $50 \mathrm{~Hz}$ to $450 \mathrm{~Hz}$ for the $Z$-axis). We show in Sec. IV-D that one of the limitations of $\mu$-path scanning is the discontinuous $X$-axis reference (e.g., in moving between $\mu$-paths) which excites the cross-coupling modes between the $X$ and $Z$ axes. We mitigate the cross coupling with a feed-forward design. While the use of such control approaches is not new, this work highlights the control challenges that arise from non-standard scanning approaches and the importance of good control design when transitioning such schemes from simulation to practice.

- We experimentally compare (Sec. VI) $\mu$-path scanning to full density raster scans and to coarse raster scans which are interpolated to a full image. The clear advantage of a coarse raster scan is that its implementation only requires adjustments to the data collection and post-processing.

- The bulk of this work is based on experiment, not simulation, in contrast to prior work by ourselves [33], [35] and others [38]-[40]. Our main focus here is on delineating the practical issues which must be addressed to effectively realize $\mu$-path scanning, which has only seen limited attention in the past.

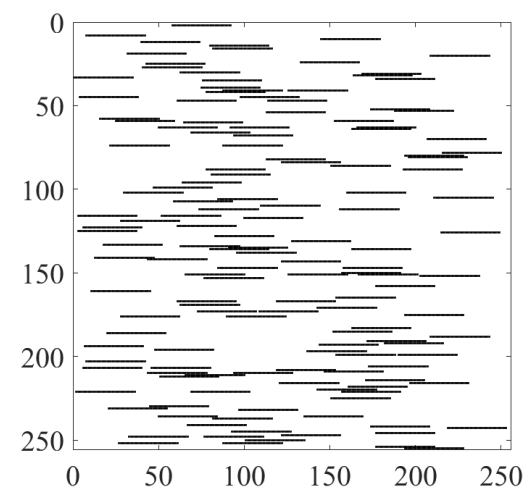

Fig. 1: An example of the horizontal $\mu$-path sampling pattern for a $256 \times 256$ pixel image with 35 pixels in each short scan and a total of $20 \%$ pixels scanned.

\section{RECONSTRUCTION METHODS}

Compressive sensing (CS) is a signal processing technique which aims at signal reconstruction from a relatively small (sub-Nyquist limit) number of measurements [41]. It takes advantage of the approximate sparsity of real-world signals, that is, that many coefficients of such signals are close to zero when represented in an appropriate basis. CS methods seek the true image signal $x \in \mathbb{R}^{n}$ from the observation equation,

$$
y=R x=R U \eta
$$

where $y \in \mathbb{R}^{m}$ is the observation vector, $R$ is an $m \times n$ matrix defining the measurements, $U$ is an $n \times n$ sparsity basis and $\eta$ is the sparse representation of $x$ in the domain of $U$. In general, $m \ll n$. In an imaging application, where the underlying signal is a matrix $X \in \mathbb{R}^{h \times s}$, we take $x=\operatorname{vec}(X)$, where the $\operatorname{vec}(\cdot)$ operator stacks the columns of a matrix.

As AFM scans are natural images, it is reasonable to expect that they satisfy the sparsity assumption and can take advantage of the reduced number of measurements needed to decrease the imaging time. An AFM probe can only measure a single pixel at a time. Thus the rows of $R$ are a subset of those of an $n \times n$ identity matrix. Ideally, the sparsity basis and the measurement matrix $R$ have a low mutual coherence, a measure of how each of the rows of $R$ (the measurements) "spreads out" in the domain of $U$ [42]. In the following, we assume that $U^{T} U=I$, and in the simulation and experimental results we make the specific choice of $U^{T}$ as the Discrete Cosine Transform (DCT). The DCT basis provides a good balance between achieving a low mutual coherence between $U$ and the $\mu$-path sensing matrices $R$ and yielding a high sparsity for typical AFM sample images.

Basis pursuit with denoising (BPDN) is a common realization of the CS-based reconstruction problem, and is given by the optimization

$$
\min _{x}\left\|U^{T} x\right\|_{1} \quad \text { subj. to } \quad\|R x-y\|_{2}<\sigma
$$

where $\sigma$ represents uncertainty in the measurements. BPDN essentially searches for the sparsest signal among the candidates that match the measurements to within the tolerance $\sigma$. 

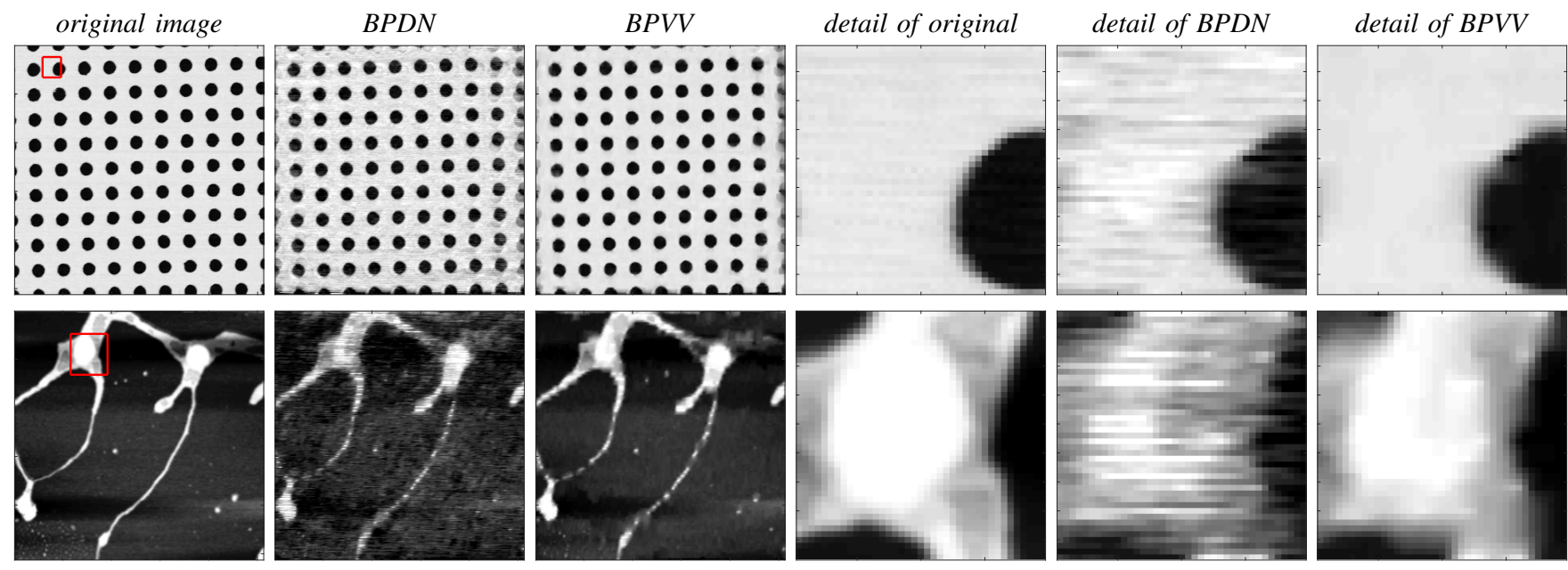

Fig. 2: Reconstruction comparison between BPDN and BPVV. (first column) Original raster-scanned image. (second column) BPDN reconstruction from random $\mu$-path sampling. (third column) BPVV reconstruction from the same sub-sampled data. (remaining columns) Corresponding details from the red boxes indicated in the raster image. The top row uses $15 \%$ sampling with 50 pixel $\mu$-paths and the bottom row uses $25 \%$ sampling with 25 pixel $\mu$-paths. The results show that BPVV reduces the artifacts arising from the horizontal scans of the $\mu$-path pattern that appear in BPDN reconstructions.

While BPDN is effective in the general setting, using it for reconstruction from horizontal $\mu$-path samples can yield artifacts in the vertical direction (orthogonal to the $\mu$-paths) leading to strong discontinuities in the image. These artifacts grow more prominent as the length of the $\mu$-paths increases [35] due to increased mutual coherence between the measurement matrix $R$ and the sparsity basis $U$. However, longer $\mu$ paths means fewer $\mu$-paths and thus shorter scan times.

To mitigate these affects, we propose a new variant of BPDN that we term basis pursuit with vertical variation (BPVV). BPVV adds a vertical total variation penalty in the spatial domain to the optimization objective, modifying (2) to

$$
\begin{aligned}
\min _{x \in Q_{p}} f(x) & =\min _{x \in Q_{p}}\left\|U^{T} x\right\|_{1}+\alpha\left\|D_{v} x\right\|_{1}+\beta\left\|D_{h} x\right\|_{1} \\
& =\min _{x \in Q_{p}}\left\|W^{T} x\right\|_{1}
\end{aligned}
$$

where

$$
W=\left[\begin{array}{lll}
U^{T} & \alpha D_{v} & \beta D_{h}
\end{array}\right]
$$

with $D_{v} x=\operatorname{vec}\left(\nabla_{v} X\right)\left(\right.$ resp., $\left.D_{h} x=\operatorname{vec}\left(\nabla_{h} X\right)\right)$ representing a discrete gradient of a matrix in the vertical (resp., horizontal) direction (see, e.g., [43, Sec. 6.1]). Thus, in (3), $\left\|D_{v} x\right\|_{1}$ (resp., $\left\|D_{h} x\right\|_{1}$ ) is the total variation (TV) of the signal in the vertical (resp., horizontal) direction. The parameters $\alpha$ and $\beta$ are weighting parameters. The description of BPVV in (4) can be interpreted as changing our assumption of sparsity in the DCT basis to assuming sparsity in an overcomplete dictionary [44]. When written as (4), the problem is in a form that may be solved with NESTA [43]. Note that the BPVV designation technically holds only for $\beta=0$; our experience, however, shows that including a small but nonzero $\beta$ (relative to $\alpha$ ) improves reconstruction. Simulation results indicate that the optimal values of $\alpha$ and $\beta$ likely depend on a variety of factors including $\mu$-path length and image type. We leave a rigorous study of these relationships to future work.
A simulation comparison between BPDN and BPVV is shown in Fig. 2. The first column shows two raster scans: the top is a $512 \times 512$ pixel image of a CS-20NG grating (Ted Pella, USA) acquired with an Agilent 5400 AFM and the bottom is a $256 \times 256$ pixel image of DNA acquired with an Agilent 5500 AFM. For the CS-20NG grating, we sampled $15 \%$ of the pixels with 50 pixel $\mu$-paths and $25 \%$ of the DNA image with 25 pixel long $\mu$-paths. The second column shows the full frame reconstructions using BPDN and the third column shows the result using BPVV (with $\alpha=0.75$ and $\beta=0.1$ ). The fourth column zooms in on the region indicated by the red box in column one. Similarly, the fifth and six columns show the detail of the reconstructions. The reconstruction results show that the artifacts apparent in the BPDN reconstruction are largely mitigated using BPVV. Thus, in the remainder of the paper, we focus exclusively on BPVV. These reconstructions with BPVV took about 1.5 seconds for the DNA image and about 7 seconds for the CS-20NG (on a typical desktop computer).

\section{EXPERIMENTAL SETUP}

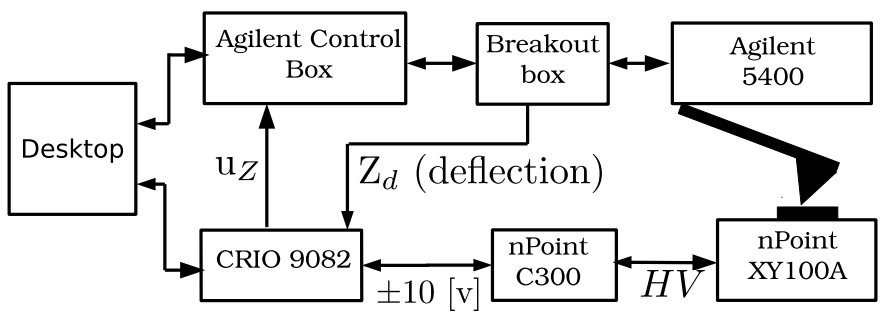

Fig. 3: Schematic diagram of the experimental AFM setup.

Our experimental setup (see Fig. 3) consists of an Agilent 5400 AFM retrofitted with an nPoint NPXY100A piezoelectric 
stage, a cRIO-9082 embedded controller (National Instruments), and a standard desktop computer. The piezoelectric tube scanner of the AFM is used only for vertical motion while the nPoint stage is used for the lateral $(X Y)$ motion.

Through a breakout box, the Agilent 5400 provides access to the $Z$-axis deflection signal. When the Agilent software is set to open-loop mode, a \pm 10 volt input on the standard control box allows control of the $Z$-axis of the N9524a piezo scanner, which has a total range of $7 \mu \mathrm{m}$. Initial (coarse) engagement of the tip to the sample is performed using the PicoView software before control is handed over to the custom controllers. All imaging is done using contact (constant force) mode, though extending to other imaging modes such as intermittent contact (tapping) mode is straightforward.

All control logic is programmed using LabVIEW 2019 and compiled to a Xilinx Spartan-6 LX150 Field Programmable Gate Array (FPGA) inside the cRIO-9082. The cRIO includes 16-bit, $100 \mathrm{kHz}$ analog-to-digital input (NI-9215) and digitalto-analog output (NI-9263) modules. All control loops are implemented using a $25 \mathrm{kHz}$ sampling rate.

\section{IMPLEMENTATION}

Implementing the $\mu$-path scheme involves operating the AFM in several distinct stages. In the $X Y$-direction, the system transitions from tracking a step command when moving to a new measurement location to tracking a short ramp during the actual $\mu$-path scan. In the $Z$-direction, the system must transition between tip descent, surface scanning, and tip retraction. Two cycles of this sequential process are illustrated by the time series in Fig. 4. The following subsections describe in more detail several features of the CS scanning process.

Transition between, and operation in, these different stages is implemented as a simple state machine, summarized in Table I. The main job of the state machine is to adjust the reference signals to accomplish each task. In the following, $r_{X}, r_{Y}$, and $r_{Z}$ refer to the references for the $X, Y$, and $Z$ axes, respectively. The $Z$-axis setpoint $r_{Z}$ takes on values $r_{Z, s}$ (the scanning setpoint) and $r_{Z \text {,up }}$ (the retraction setpoint).

TABLE I: Summary of the tasks for $\mu$-path scanning.

\begin{tabular}{lccc} 
state & $r_{X}, r_{Y}$ & $r_{Z}$ & next-state \\
\hline (1) $X Y$-move & setpoint & $r_{Z}=r_{Z \text {,up }}$ & (2) \\
(2) tip-engage & setpoint & $r_{Z}=r_{Z, \mathrm{~s}}$ & (3) \\
(3) pre-scan & ramp & $r_{Z}=r_{Z, \mathrm{~s}}$ & (4) \\
(4) $\mu$-path scan & ramp & $r_{Z}=r_{Z, \mathrm{~s}}$ \\
(5) tip up & setpoint & $r_{Z}=r_{Z \text {,up }}$
\end{tabular}

\section{A. Cantilever setpoint during the $X Y$-move}

In order to minimize scanning time, the tip should be moved as fast as possible when transitioning between $\mu$-path locations (state (1)). Since such speeds can be beyond the bandwidth of the $Z$-axis controller, it is important to reduce the tip-sample interaction force to prevent tip wear and sample damage. In our initial implementation [37], this was achieved by insisting that during tip-retraction (state (5)), the cantilever tip should fully break contact with the surface. Here, we impose no such requirement. Rather, we change the $Z$-setpoint
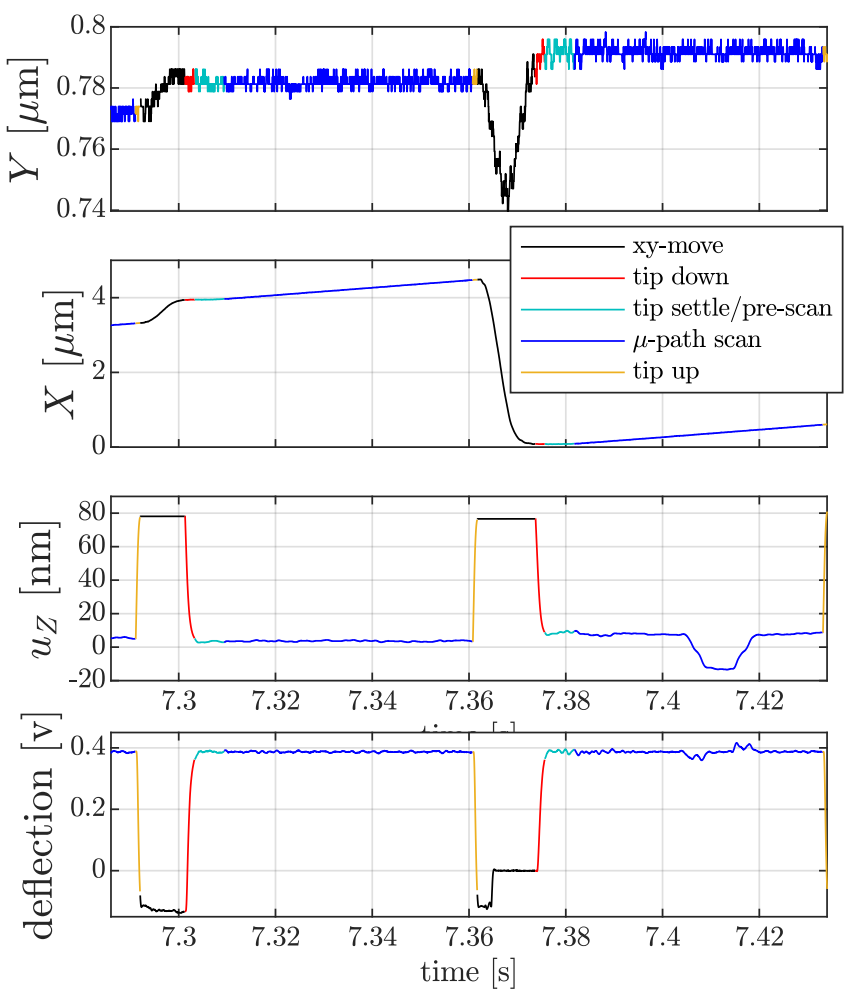

Fig. 4: Several CS cycles. Each state is indicated by color. The deflection is shifted so its free value is zero.

during tip-retraction to only pull away far enough that the deflection signal stays low (i.e., below the scanning setpoint), even while we run across the surface. We choose $r_{Z \text {, up }}$ so that the interaction between the tip and sample becomes purely adhesive. In general, stable imaging is not possible at this setpoint and complete dis-engagement occasionally occurs. This can be seen in the bottom pane of Fig. 4 during the second $X Y$-move (black portion) where the tip detaches partway through the move. When dis-engagement occurs, the $Z$-axis control loop implements an anti-windup scheme to prevent the control from saturating. In our implementation, we programmatically stop the accumulator from accumulating while dis-engagement is detected.

Only moving the tip into the adhesive region during the $X Y$ move saves considerable time compared to completely detaching the tip between each $\mu$-path. Selecting a $Z$-setpoint lower than the scanning setpoint ensures very low normal forces. However, it is important to note that with the tip even lightly engaged, the move to the new scanning location may cause shear forces which may be a challenge for some biological samples. If necessary, the tip can be completely disengaged, though at a cost in imaging time. Completely characterizing the damage done to the tip and sample with this scheme compared to complete detachment (which can also damage the probe [45]), remains an outstanding goal.

\section{B. The pre-scan}

After the tip-descent, we begin scanning in a "pre-scan" phase (state (3)), which is the turquoise "tip settle" portion of 


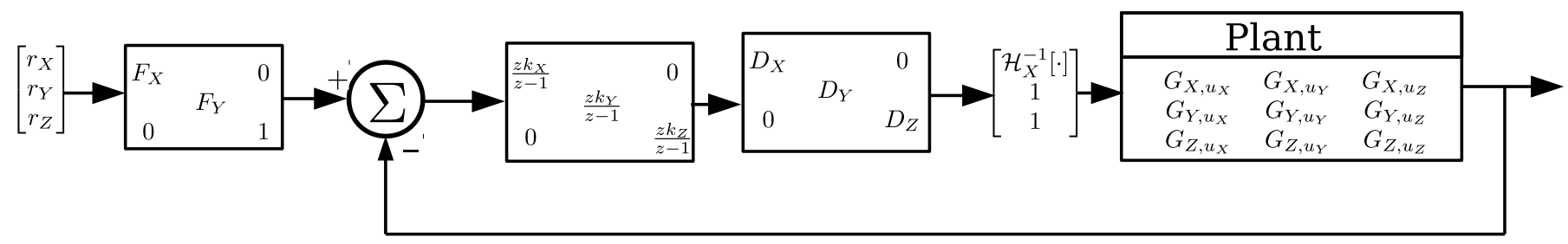

Fig. 5: Block diagram of the AFM control loop for all three axes. Not shown is anti-windup for the $Z$-axis integrator, which is active when $r_{Z}=r_{Z \text {,up }}$ (states (1) and (5)) where sensor saturation is possible.

the trajectories in Fig. 4. There are two reasons for this. First, we have observed that there is less noise in the deflection signal while the $X Y$-stage is in motion compared to sitting still. Second, and more importantly, it lets us bring the $X$-axis to its scanning velocity at the same time the transients from the $Z$-axis die out. Without this, we must extend the $\mu$-path by a distance equal to the steady-state error following a ramp. For an arbitrary scan speed, this means the $\mu$-path scan would need an extra

$$
N=\left\lceil\lim _{z \rightarrow 1} \frac{1-H_{X}}{z-1}\right\rceil
$$

time steps, where $H_{X}$ is closed-loop transfer function for the $X$-axis. For our system $N=111$. By initiating the scan while the $Z$-axis settles, we eliminate much of this overhead.

\section{Control}

The control structure for each axis, shown in the block diagram in Fig. 5, is similar and essentially consists of a set of filters in series with a PI controller. The open-loop frequency responses for the $X$ and $Y$ axes are shown as the black curves in Fig. 6a and for the $Z$-axis in Fig. 6c. The first resonance of each axis is the bending mode, which appears at $215 \mathrm{~Hz}$ for the $Z$ axis and about $350 \mathrm{~Hz}$ for the $X$ and $Y$ axes.

The filter portion of the $Z$-axis controller $D_{Z}$ is secondorder and simply inverts the complex-conjugate pole-zero pair at $215 \mathrm{~Hz}$. This allows the gain of the PI controller to be increased substantially. The closed-loop achieves a bandwidth of about $450 \mathrm{~Hz}$, as shown by the red curve in Fig. 6c. Without this inversion, the bandwidth is limited to about $50 \mathrm{~Hz}$.

We take a similar approach for the $X$ and $Y$ axes. The filters $D_{X}$ and $D_{Y}$ invert the bending modes at $350 \mathrm{~Hz}$ and add notch filters at the main resonant peaks near $650 \mathrm{~Hz}$. The resulting closed-loop transfer functions are shown as the red curves in Fig. 6a.

In general, the $X$-axis positioning requirements are more stringent for both CS and raster scanning. Due to the step inputs the $X$-axis must track, we have found that compensating hysteresis and creep to be particularly helpful during $\mu$-path scanning. Thus, the $X$-axis compensator also includes an inverse creep and hysteresis model. The hysteresis model is a modified Prandtl-Ishlinskii type and is based on the work of [46]. The drift compensation models the piezo creep as a simple second-order transfer function with real poles and zeros whose inverse is part of $D_{X}$. In Fig. 5, the hysteresis inversion is indicated by $\mathcal{H}_{X}^{-1}[\cdot]$. We have found that the inclusion of these extra compensators limits the overshoot of the $X$-axis during larger moves between different $\mu$-paths (e.g., without them, the $X$-axis trajectory in Fig. $6 \mathrm{~b}$ would show noticeable overshoot). More details on fitting the creep and hysteresis models can be found in our other work [47].

\section{Feedforward Control}

As the bottom row of plots in Fig. 6a makes clear, there is a strong cross-coupling from the horizontal plane to the $Z$ axis (the coupling from $u_{Z}$ to the $X$ and $Y$ axes is very weak, and so is not shown). Our SISO control scheme does little to attenuate the resonances of $G_{Z, u_{X}}$ and $G_{Z, u_{Y}}$. In particular, there are modes in both these transfer functions at 215 and 505 $\mathrm{Hz}$ that do not appear in a SISO model of $G_{X, u_{X}}$ or $G_{Y, u_{Y}}$.

For raster scans, the frequency content of the $X$-axis reference triangle wave decays like $1 / n^{2}$ with a gain proportional to the raster frequency. In contrast, the $X$-axis reference for a $\mu$-path scan is composed of a sequence of steps and ramps. The inclusion of the (discontinuous) steps means we should expect the $\mu$-path $X$ reference to decay like $1 / n$. Thus, while the cross-coupling can induce $Z$-axis vibrations for faster raster scans, it is especially problematic for $\mu$-path scanning. This is illustrated in the left column of Fig. 6b, which shows a CS cycle during which the $X$-axis makes a $5 \mu \mathrm{m}$ move, which induces excessively large vibrations in the vertical control.

In principle, one could mitigate these issues with a full MIMO control design. In this work, we opt for a much simpler feedforward scheme. For CS scanning, we insert feed-forward filters ( $F_{X}$ and $F_{Y}$ in Fig. 5) with three notches: at $215 \mathrm{~Hz}$, $350 \mathrm{~Hz}$, and $505 \mathrm{~Hz}$. Both filters are shown in Fig. 6a as the dashed-pink curves. The resulting overall FRF, shown as the blue curves, show a dramatic reduction in the cross coupling. A similar effect could be achieved by using a simple low-pass filter. However, to achieve the same $40 \mathrm{~dB}$ of attenuation of the mode at $215 \mathrm{~Hz}$ would require, e.g., that a two-pole low-pass filter have its cut-off frequency at about $20 \mathrm{~Hz}$. Thus, while our feedforward scheme slows the $X$-axis down, we achieve better bandwidth than we would with a simple low-pass filter.

For raster scanning, we set $F_{X}=F_{Y}=1$ and instead take advantage of the periodicity of the triangle-wave $X$ axis reference. We modify the triangle wave to a truncated Fourier series, where each Fourier coefficient is scaled by the inverse of the closed-loop FRF at the corresponding frequency. See, e.g., [48] for more details on this strategy. We truncate this series such that the highest frequency is smaller than $200 \mathrm{~Hz}$, which means that we should never excite the problematic $G_{Z, u_{X}}$ modes. This scheme implies that the effective bandwidth of the $X$-axis controller is higher while raster scanning than while CS-scanning. On the other hand, it 


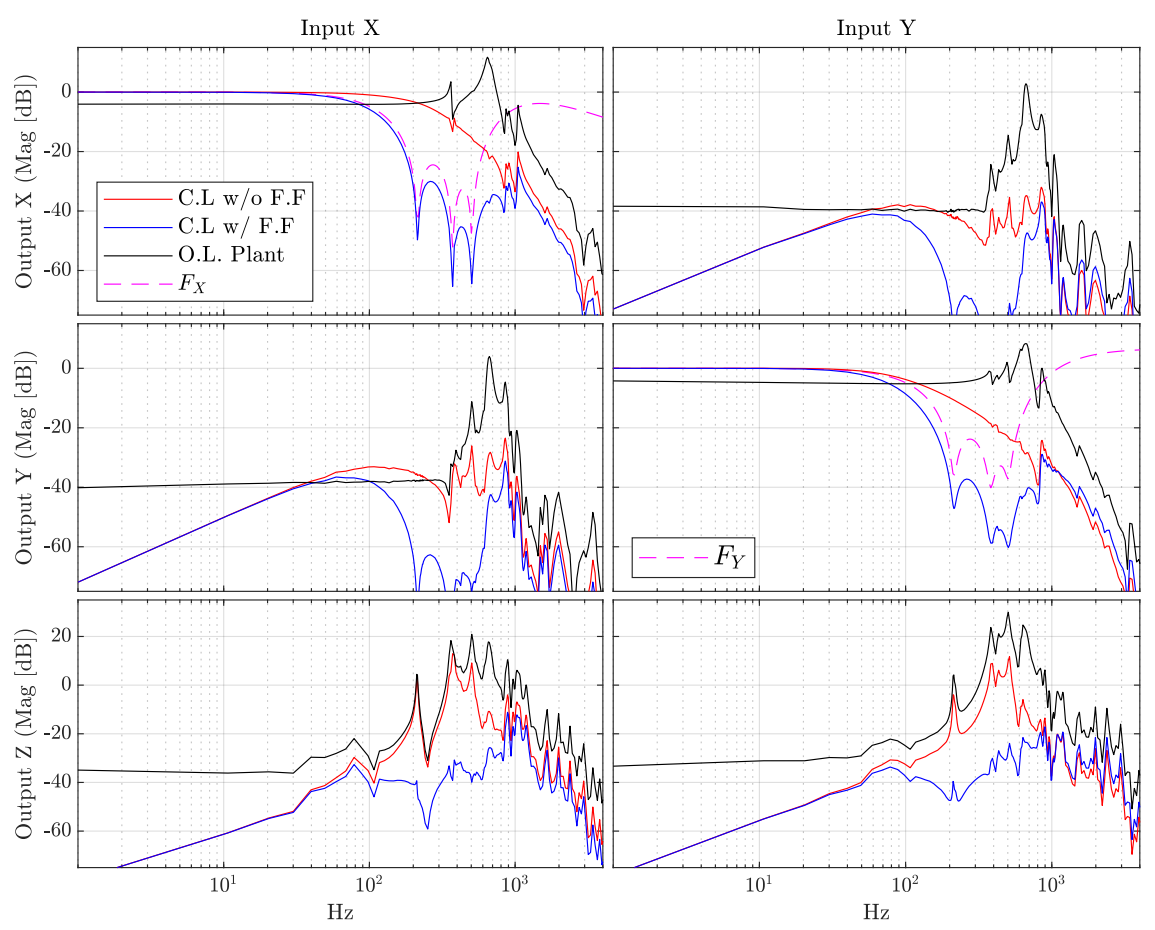

(a)

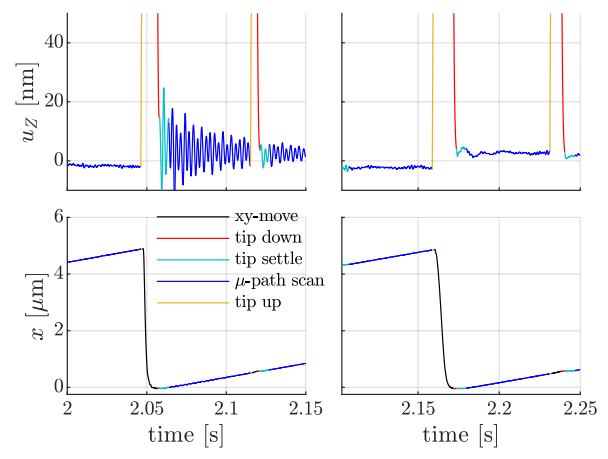

(b)

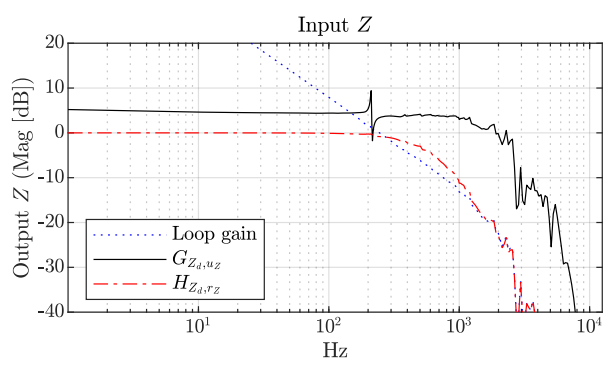

(c)

Fig. 6: (a) MIMO closed and open-loop frequency-response functions (FRFs), showing the dramatic effect on $G_{Z, u_{X}}$ and $G_{Z, u_{Y}}$ when the feedforward compensators are used for the $X$ and $Y$ axes. (b) The vertical control $u_{Z}$ (top) after a large move in the $X$-direction (bottom) without (left column) and with (right column) the feedforward compensation. (c) Frequency response of the $Z$-axis in open-loop (black) and closed-loop (red).

also implies that for faster scan rates, fewer Fourier coefficients are used and the triangle-wave approximation is worse, leading to scans which cover slightly less than the intended range.

\section{METRICS}

In this section we introduce the three metrics used to assess our results in Sec. VI. The first two seek to quantify image quality between two images. To that end, define a master image as $X$ and a reconstructed or corrupted version as $Y$, with each having $s \times h=m$ pixels. Let $x=\operatorname{vec}(X)$, $y=\operatorname{vec}(Y)$, and $L$ be the dynamic range of the master image $x$. The first metric is the peak signal-to-noise ratio (PSNR):

$$
\operatorname{PSNR}(x, y)=10 \log _{10} \frac{L^{2}}{\sqrt{\frac{1}{m} \sum_{i=1}^{m}\left(x_{i}-y_{i}\right)^{2}}} .
$$

The second metric is the Structured Similarity Index Metric (SSIM). The goal of the SSIM is to compare the structure, luminescence, and contrast of two images. It is built up from the means $\left(\mu_{x}\right.$ and $\left.\mu_{y}\right)$, standard deviations $\left(\sigma_{x}\right.$ and $\left.\sigma_{y}\right)$, and covariance $\left(\sigma_{x y}\right)$ of the image vectors $x$ and $y$. The SSIM definition used in this paper is

$$
\operatorname{SSIM}(x, y)=\frac{\left(2 \mu_{x} \mu_{y}+C_{1}\right)\left(2 \sigma_{x y}+C_{2}\right)}{\left(\mu_{x}^{2}+\mu_{y}^{2}+C_{1}\right)\left(\sigma_{x}^{2}+\sigma_{y}^{2}+C_{2}\right)}
$$

where the constants $C_{1}$ and $C_{2}$ are regularizing constants to prevent singularity if, e.g., $\mu_{x}=\mu_{y}=0$. We use the default values suggested in [49] of $C_{1}=(0.01 L)^{2}$ and $C_{2}=(0.03 L)^{2}$, where again, $L$ is the dynamic range. In general two images which are identical will yield an infinite PSNR and an SSIM of 1.

\section{A. Limitations of SSIM and PSNR}

The SSIM and PSNR have become reasonably popular in studies comparing simulated AFM image reconstructions [33], [38], [40]. However, it remains somewhat of an open question how to best compare experimental AFM images. While we use the SSIM and PSNR in Sec. VI, we believe those numbers should be interpreted with some caution. In our experimental setup, actuation in the $X Y$-plane happens via the nPoint piezo stage and the $X Y$-direction of the original piezo tube scanner is uncontrolled. This leads to substantial drift between images. To illustrate this, we took a sequence of 6 raster scans of a sample grating, each at a $1 \mathrm{~Hz}$ scan rate over a $5 \mu \mathrm{m} \times 5 \mu \mathrm{m}$ area. The left image of Fig. 7 shows the error between the first and sixth image. The PSNR and SSIM between the first and second images are 15.08 and 0.51 while between the first and sixth they are 8.49 and 0.26. Despite this, on their own each image appears to have good quality.

To mitigate this effect, we use an image alignment technique based on the 2D cross-correlation between each image. Essentially, this takes a sub-slice of the master (first) image (here, a 25 pixel inset on each edge), and finds the offset in the other image that produces the smallest error. The result of this procedure is shown in the right image of Fig. 7. Although the situation is improved compared to the un-aligned images, there is still noticeable stretching (the upper and lower portions 


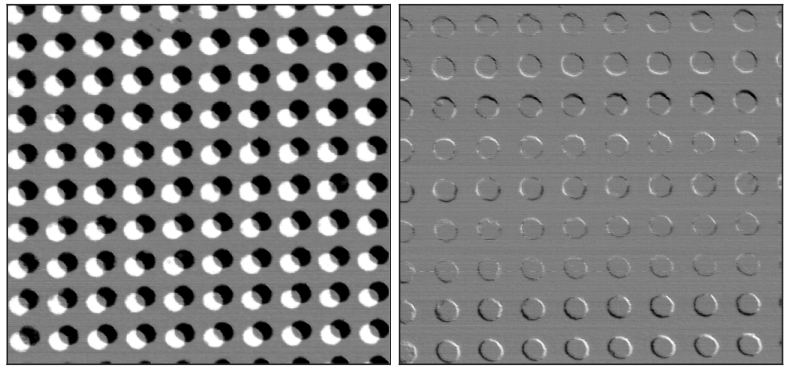

Fig. 7: Errors between the first and sixth raster scans in a sequence of six $1.0 \mathrm{~Hz}$ raster scans. (left) Errors without alignment. (right) The same data but aligned via cross correlation.

appear considerably worse than the middle). Notably however, with the alignment technique, the SSIM is 0.72 for both the second and sixth image images and the PSNR increases to 23.40 and 22.68 , respectively.

\section{$B$. The relative damage index}

The PSNR and SSIM metrics only assess the similarity of two images. However, in AFM imaging, pure image quality is not the only concern, particularly for delicate samples. While damage to the specimen is not really a concern while imaging a hard calibration grating, it plays a prominent role for biological samples [50], though damage to the probe itself is still a concern even with hard samples. In general, the faster the scan rate, the more difficulty the $Z$-axis control will have in following the sample surface, which results in a larger deflection signal $Z_{d}$ over more time. The actual damage done by a given $Z_{d}$ will depend on the spring constant of the cantilever and the softness of the specimen. To capture these concerns, we propose a metric, which we call the Relative Damage Index (RDI), that computes the power in the deflection signal for a given scan. The RDI, given by

$$
\mathrm{RDI}=\frac{1}{T_{s}|I|} \sum_{k \in I}\left(Z_{d, k}-r_{Z, s}\right)^{2},
$$

with $I=\left\{k: r_{Z, k}=r_{Z, s}\right\}$ and $T_{s}$ the sample period, accumulates the deflection signal while scanning as a measure of the net force applied to the specimen over time.

\section{EXPERIMENTAL RESULTS}

We compared $\mu$-path scanning to two scenarios: first to full resolution raster scans at several scan rates in Sec. VI-A, and second, to coarse raster scans in Sec. VI-B.

\section{A. Comparison to full raster}

We imaged a $5 \mu \mathrm{m} \times 5 \mu \mathrm{m}$ area of a sample grating with $20 \mathrm{~nm}$ deep holes on a $500 \mathrm{~nm}$ pitch (Ted Pella, CS-20NG). Standard raster scans with 512 lines were performed at $1.0 \mathrm{~Hz}$, $2.5 \mathrm{~Hz}, 5.0 \mathrm{~Hz}$, and $8.0 \mathrm{~Hz}$. We also took $\mu$-path scans with designed densities of $12.5 \%$ and $25 \%$. The same $\mu$-paths were used throughout the experiments. $512 \times 512$ pixel images were reconstructed using BPVV with $\alpha=0.75$ and $\beta=0.1$. The
TABLE II: State times (in seconds) for the CS scans in Fig. 8

\begin{tabular}{ccccccc} 
description & move & engage & pre-scan & scan & tip-up & total \\
\hline $1.0 \mathrm{~Hz}, 12.4 \%$ & 6.16 & 1.13 & 3.82 & 32.25 & 0.24 & 43.60 \\
$5.0 \mathrm{~Hz}, 12.4 \%$ & 6.09 & 1.01 & 3.82 & 6.73 & 0.08 & 17.73 \\
$8.0 \mathrm{~Hz}, 12.7 \%$ & 6.14 & 1.02 & 3.82 & 4.35 & 0.08 & 15.40 \\
$1.0 \mathrm{~Hz}, 24.0 \%$ & 11.84 & 2.40 & 7.62 & 64.41 & 0.15 & 86.42 \\
$5.0 \mathrm{~Hz}, 25.3 \%$ & 11.54 & 2.19 & 7.62 & 13.44 & 0.53 & 35.32 \\
$8.0 \mathrm{~Hz}, 24.8 \%$ & 11.73 & 2.06 & 7.62 & 8.68 & 0.15 & 30.25
\end{tabular}

CS scans were taken with scan velocities equivalent to raster scan rates of $1.0 \mathrm{~Hz}, 2.0 \mathrm{~Hz}, 4.0 \mathrm{~Hz}, 5.0 \mathrm{~Hz}$ and $8.0 \mathrm{~Hz}$. For all CS scans, the $\mu$-paths were $500 \mathrm{~nm}$ long, which translates to 51 pixels. For each image, the $\mu$-paths were scanned in a left-to-right, bottom-to-top fashion.

The free value of the deflection signal was about -0.6 volts. The scanning reference was set to $r_{Z, \mathrm{~s}}=-0.3$ volts (for both raster and CS) and the withdraw reference to $r_{Z \text {,up }}=-0.8$ volts. We set the $X Y$-settle boundary to $\pm 0.02 \mu \mathrm{m}$ and required 20 samples within this boundary before moving to the $Z$-engage state. The pre-scan length was 150 time steps. The cantilever was a Budget Sensors ContAI-G, with nominal length of $450 \mu \mathrm{m}$ and spring constant of $0.2 \mathrm{~N} / \mathrm{m}$.

For the raster scans, we discarded data from the re-trace and divided the remaining data for each scan line into 512 bins based on the $X$ sensor measurements. Data in each bin were averaged to obtain the value of one pixel. To remove the effects of piezo creep and sample tilt, we de-trended each individual line. De-trending each line causes the flat portion between the holes in the horizontal direction to appear at a different height than the flat portion between holes in the vertical direction. To remove this effect, we selected a column of pixels on the left and right side of each image that did not cross any holes (i.e., a vertical line through the flat area), and registered each scan line to a common height along these columns. This step eased comparison with the CS scans and was inspired by a feature in the AFM image processing toolbox SPIW [51]. A subset of the resulting images are shown in Fig. 8. The rows of pixels indicated by the red lines are shown as cross sections in Fig. 9. For the CS scans shown in Fig. 8, Table II breaks down the amount of time spent on each task.

Qualitatively, the CS images are of high quality despite the significantly lower number of measurements, though the reconstructions from BPVV do show a small amount of blurring at their edges. For a quantitative comparison, we computed the SSIM and PSNR for each raster and CS scan, using the $1 \mathrm{~Hz}$ raster scan as the master and also computed the RDI metric. These are plotted against total acquisition time in Fig. 10. Roughly speaking, image quality improves as acquisition time increases. However, the metrics are quite noisy and the results of the different methods are overlapping, making it difficult to extract a general result on the scanning approaches.

Things are clearer if we plot the RDI for each scan against total acquisition time, shown in Fig. 10c. As one would expect, as imaging time decreases, the RDI for each scan type increases. From Fig. 10c, we see that for a fixed RDI, CS has a faster imaging acquisition rate. Conversely, for an acquisition time below a given threshold, CS offers a smaller RDI than 


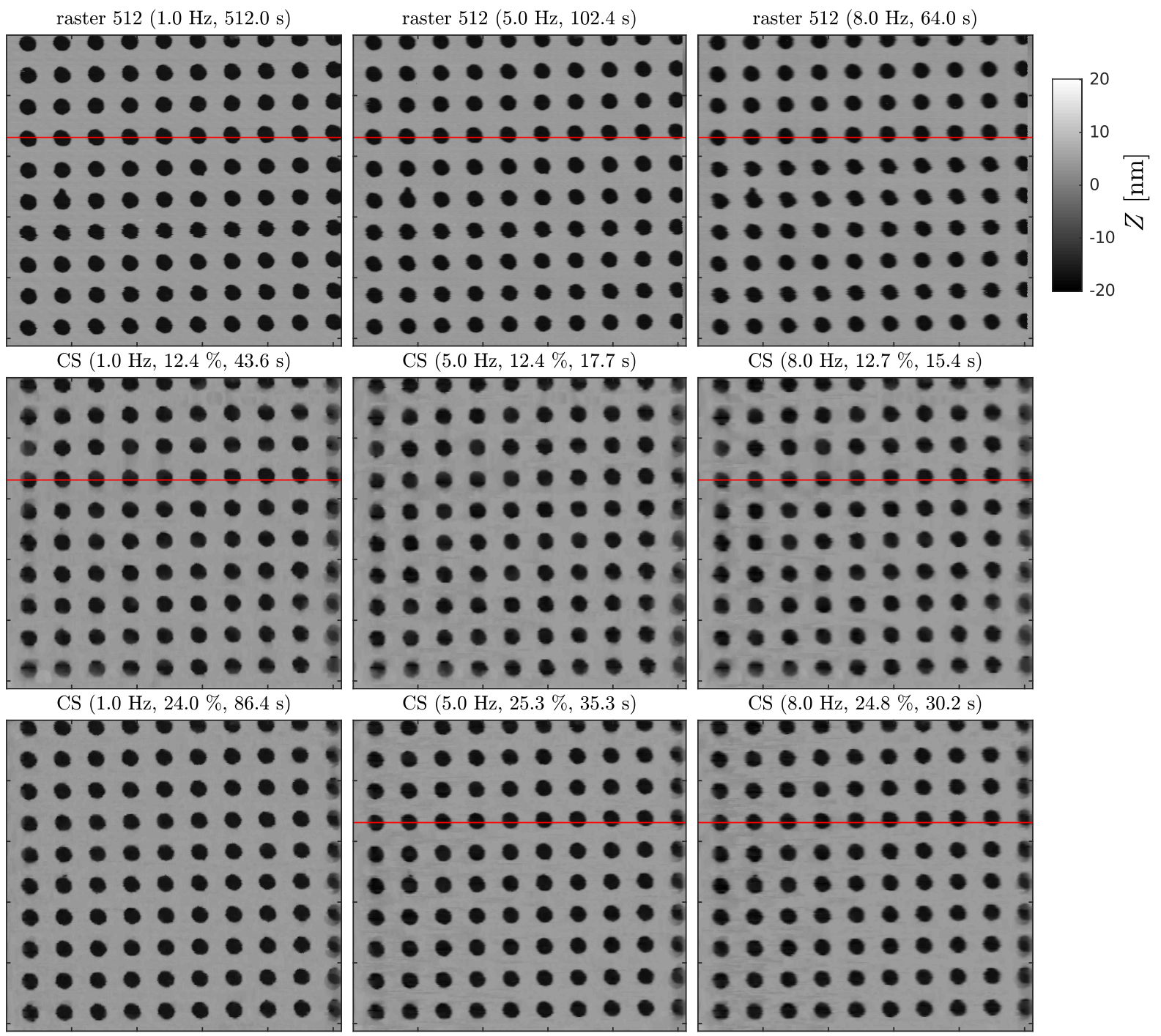

Fig. 8: Raster and compressed sensing images of a 5 micron by 5 micron area of the CS-20NG grating. All images are $512 \times 512$ pixels. The rows of pixels indicated by the red lines are shown in Fig. 9.
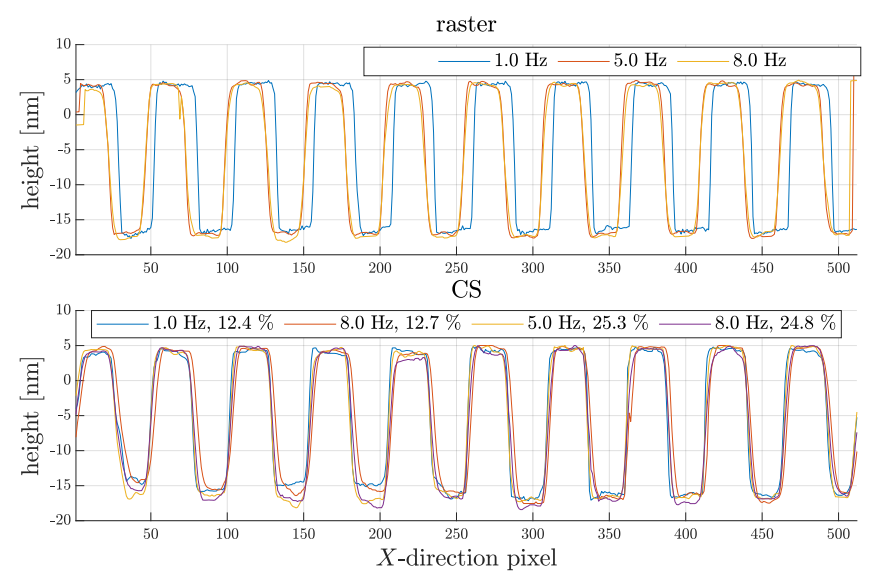

Fig. 9: Rows of pixels, as indicated by the red lines in Fig. 8. For clarity, not all images are included. a full raster scan. The amount of improvement is best if we need a very low RDI: for example, if we want to hold the RDI below 2, then we have to take the $1 \mathrm{~Hz}$ raster scan (512 seconds) while we can achieve the same RDI via CS in about 42 seconds with the $12.5 \%$ sampling, a speed improvement of over a factor of 10 . However, the advantage of CS over raster narrows as the allowable RDI is increased as a result of the constant overhead imposed by the engage/disengage and $X Y$ move states in the CS scans. For example, the $8 \mathrm{~Hz}$ raster scan and $8 \mathrm{~Hz}, 12.5 \% \mathrm{CS}$ scan have comparable RDIs, but now CS is only about 5 times faster.

\section{B. Comparison to subline sampling}

Many subline sampling papers assume that the lines to sample are selected randomly [33], [38]. An even simpler alternative to $\mu$-path sampling is to take a standard raster scan with fewer lines. Here we consider scanning images with 128 and 64 lines. In each case, the data for each line is first divided into 512 bins to yield data that is 128 (resp., 64) pixels by 


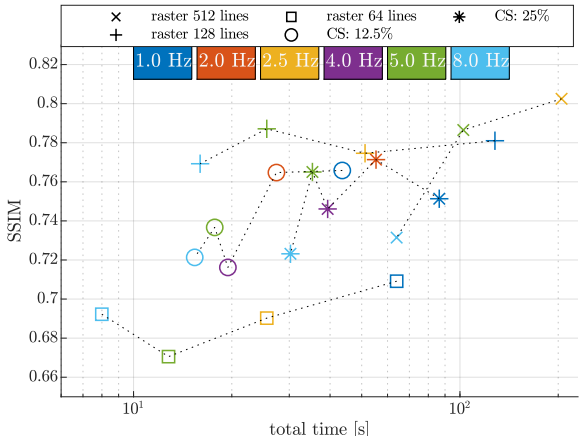

(a)

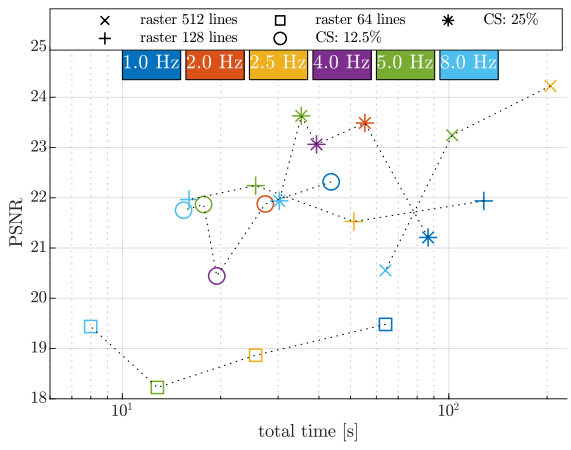

(b)

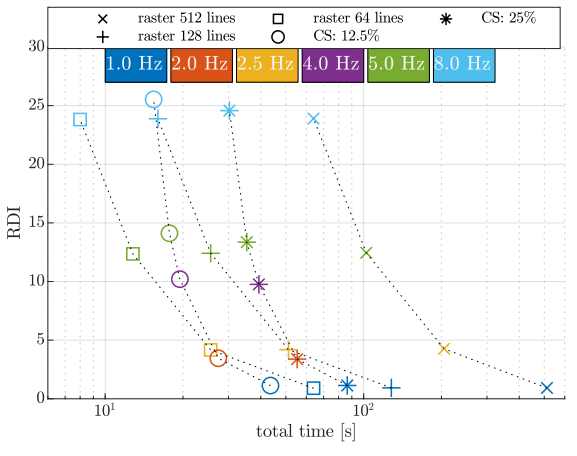

(c)

Fig. 10: Metrics for raster scans at 512, 128, and 64 lines and $\mu$-path scans at $12.5 \%$ and $25 \%$ sampling. The scan rate is indicated by color while the scan style is indicated by marker style. (a) SSIM as a function of total acquisition time. (b) PSNR as a function of total acquisition time. (c) RDI as a function of total acquisition time.

512 pixels, corresponding to $25 \%$ (resp., $12.5 \%$ ) sampling. This data is then linearly interpolated along each column to produce a $512 \times 512$ pixel image. As before, these scans were performed at 1.0, 2.5, 5.0, and $8.0 \mathrm{~Hz}$. A subset of the resulting images are shown in Fig. 11.

Both visually from Fig. 11 and the SSIM and PSNR metrics shown in Figs. 10a and 10b, the 64 line scan performs the worst of all sub-sampling methods and is slower than $12.5 \%$ CS for slow scan rates. However, the 128 line scans appear to have as good or better image quality than either CS scans.

Fig. 12 compares the speed improvement of the different sub-sampling schemes over a full raster scan with 512 lines as a function of scan rate. The $12.5 \% \mathrm{CS}$ scan at $1 \mathrm{~Hz}$ shows the most improvement, though the 64 line raster scan is close behind. Due to the constant overhead imposed by the engage/disengage and $X Y$-move states in the CS scans, the relative gain of the CS approach diminishes at faster scan rates and eventually the 64- and 128-line scans are faster. In general, the artifacts of the CS reconstructions are different than the interpolated raster scans. CS appears to preserve the edges of the holes better than subline sampling, while subline sampling has less noise in the flat areas of the specimen.

\section{CONCLUSIONS}

We have introduced a new reconstruction method designed to reduce $\mu$-path sampling artifacts. We then addressed several practical implementation issues. Chief among these is the need to more aggressively mitigate coupling between the horizontal plane and the $Z$-axis than is required for raster scanning. Here, we applied a feedforward control scheme which attenuates the problematic modes by approximately $40 \mathrm{~dB}$.

We then experimentally demonstrated $\mu$-path sub-sampling and compared the resulting images to both full raster scans and coarse raster scans paired with reconstruction. The main conclusion of this comparison is that with $\mu$-paths, we can acquire images faster yet with less specimen damage than is possible while raster scanning a full image and that for slow scan rates, $\mu$-path scanning achieves better images in less time than subline sampling. On the other hand, for faster scan rates (and especially with higher sampling densities), reconstructing a coarse raster scan makes more sense. This is notable, since a coarse raster scan with reconstruction is considerably easier to implement, both on the hardware side as well as the postprocessing side. It remains to be seen if this conclusion holds for a wide range of instruments and specimens.

\section{REFERENCES}

[1] D. Y. Abramovitch, S. B. Andersson, L. Y. Pao, and G. Schitter, "A tutorial on the mechanisms, dynamics, and control of atomic force microscopes," in Proc. American Control Conference, 2007, pp. 34883502.

[2] Y. F. Dufrêne, T. Ando, R. Garcia, D. Alsteens, D. Martinez-Martin, A. Engel, C. Gerber, and D. J. Muller, "Imaging modes of atomic force microscopy for application in molecular and cell biology," Nature Nanotechnology, vol. 12, no. 4, pp. 295-307, Apr. 2017.

[3] C. Yang, R. Winkler, M. Dukic, J. Zhao, H. Plank, and G. E. Fantner, "Probing the morphology and evolving dynamics of 3D printed nanostructures using high-speed atomic force microscopy," ACS Applied Materials \& Interfaces, vol. 9, no. 29, pp. 24 456-24 461, July 2017.

[4] O. D. Payton, L. Picco, and T. B. Scott, "High-speed atomic force microscopy for materials science," International Materials Reviews, vol. 61, no. 8, pp. 473-494, June 2016.

[5] E. I. Altman, M. Z. Baykara, and U. D. Schwarz, "Noncontact atomic force microscopy: An emerging tool for fundamental catalysis research," Accounts of Chemical Research, vol. 48, no. 9, pp. 2640-2648, Aug. 2015.

[6] K. Haase and A. E. Pelling, "Investigating cell mechanics with atomic force microscopy," Journal of the Royal Society Interface, vol. 12, no. 104, p. 20140970 (16 pages), Mar. 2015.

[7] M. Shibata, H. Nishimasu, N. Kodera, S. Hirano, T. Ando, T. Uchihashi, and O. Nureki, "Real-space and real-time dynamics of CRISPR-Cas9 visualized by high-speed atomic force microscopy," Nature Communications, vol. 8, no. 1, p. 1430, Nov. 2017.

[8] M. Shibata, T. Uchihashi, T. Ando, and R. Yasuda, "Long-tip high-speed atomic force microscopy for nanometer-scale imaging in live cells," Scientific Reports, vol. 5, no. 1, p. 8724, Mar. 2015.

[9] T. Ando, T. Uchihashi, and S. Scheuring, "Filming biomolecular processes by high-speed atomic force microscopy," Chemical Reviews, vol. 114, no. 6, pp. 3120-3188, Jan. 2014.

[10] M. B. Viani, T. E. Schaeffer, G. T. Paloczi, L. I. Pietrasanta, B. L. Smith, J. B. Thompson, M. Richter, M. Rief, H. E. Gaub, K. W. Plaxco, A. N. Cleland, H. G. Hansma, and P. K. Hansma, "Fast imaging and fast force spectroscopy of single biopolymers with a new atomic force microscope designed for small cantilevers," Review of Scientific Instruments, vol. 70, no. 11, pp. 4300-4303, 1999.

[11] C. Braunsmann and T. E. Schäffer, "High-speed atomic force microscopy for large scan sizes using small cantilevers," Nanotechnology, vol. 21, no. 22, p. 225705, 2010.

[12] J. D. Adams, B. W. Erickson, J. Grossenbacher, J. Brugger, A. Nievergelt, and G. E. Fantner, "Harnessing the damping properties of materials for high-speed atomic force microscopy," Nature Nanotechnology, vol. 11, no. 2, pp. 147-151, Feb. 2016. 
raster $128(1.0 \mathrm{~Hz}, 128.0 \mathrm{~s})$

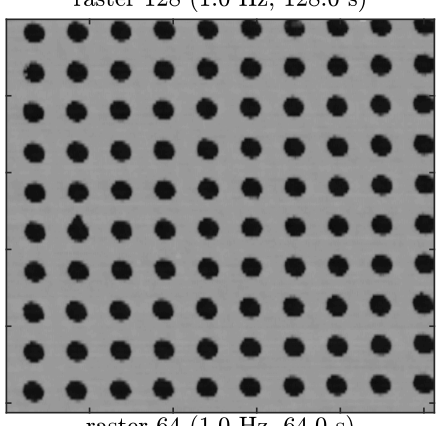

raster $64(1.0 \mathrm{~Hz}, 64.0 \mathrm{~s})$

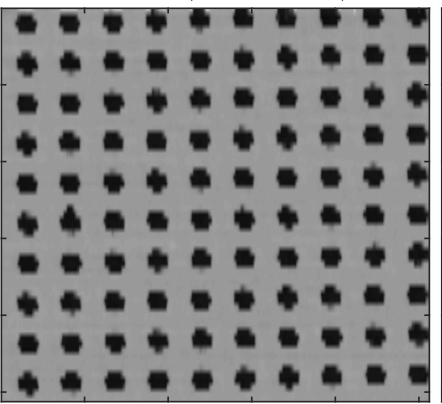

raster $128(5.0 \mathrm{~Hz}, 25.6 \mathrm{~s})$

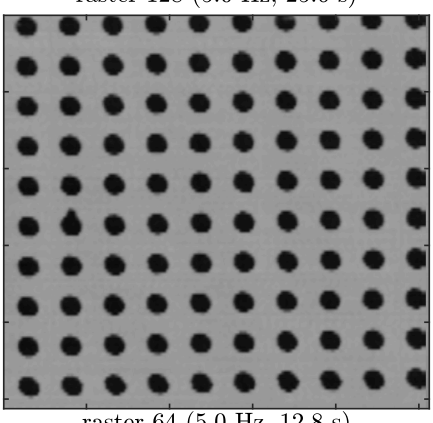

raster $64(5.0 \mathrm{~Hz}, 12.8 \mathrm{~s})$

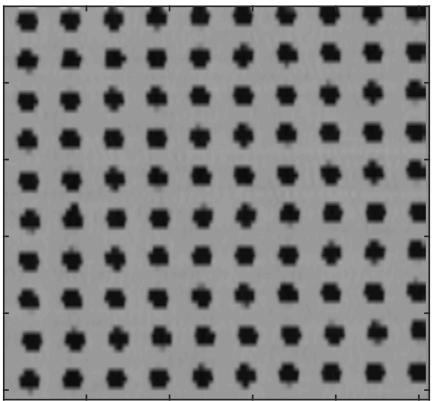

raster $128(8.0 \mathrm{~Hz}, 16.0 \mathrm{~s})$

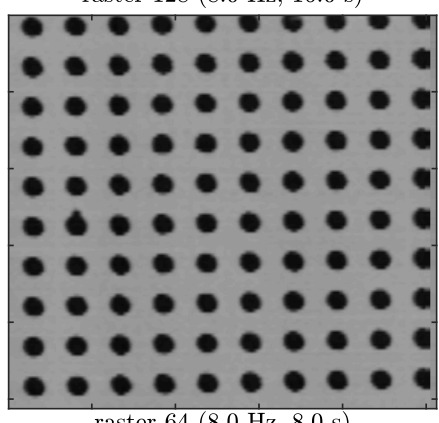

raster $64(8.0 \mathrm{~Hz}, 8.0 \mathrm{~s})$

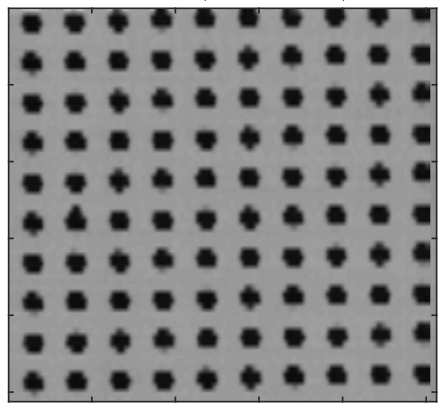

Fig. 11: Raster scans of 128 (top) and 64 (bottom) lines which have been interpolated along each column to 512 lines.

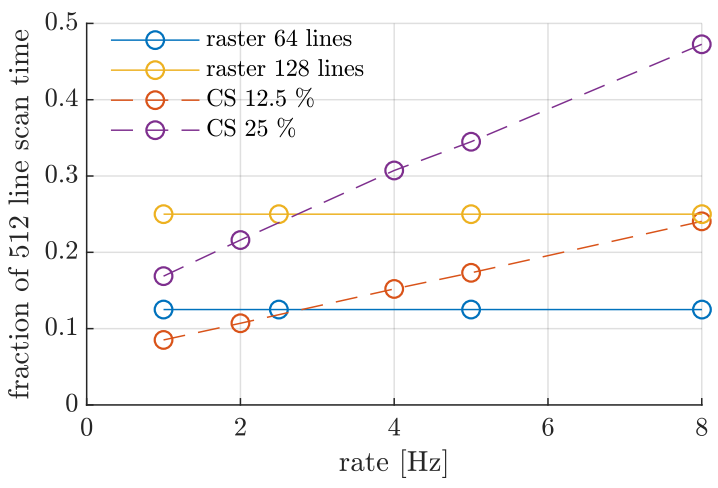

Fig. 12: Fraction of improvement in total scan time over a scan with 512 lines vs scan rate for the two sets of CS scans (at $25 \%$ and $12.5 \%$ ) and coarse raster scans at 128 and 64 lines.

[13] M. Maroufi, A. Bazaei, and S. O. R. Moheimani, "A high-bandwidth MEMS nanopositioner for on-chip AFM: Design, characterization, and control," IEEE Transactions on Control Systems Technology, vol. 23, no. 2, pp. 504-512, Mar. 2015

[14] Y. K. Yong, S. O. R. Moheimani, B. J. Kenton, and K. K. Leang, "Invited review article: High-speed flexure-guided nanopositioning: Mechanical design and control issues," Review of Scientific Instruments, vol. 83, no. 12, p. 121101 (22 pages), Dec. 2012.

[15] B. J. Kenton and K. K. Leang, "Design and control of a three-axis serial-kinematic high-bandwidth nanopositioner," IEEE Transactions on Mechatronics, vol. 17, no. 2, pp. 356-369, Apr. 2012.

[16] M. S. Rana, H. R. Pota, and I. R. Petersen, "A survey of methods used to control piezoelectric tube scanners in high-speed AFM imaging," Asian Journal of Control, vol. 20, no. 4, pp. 1379-1399, July 2018.

[17] Y. K. Yong and S. O. R. Moheimani, "Collocated z-axis control of a high-speed nanopositioner for video-rate atomic force microscopy," IEEE Transactions on Nanotechnology, vol. 14, no. 2, pp. 338-345, Mar. 2015.

[18] J. A. Butterworth, L. Y. Pao, and D. Y. Abramovitch, "A discretetime single-parameter combined feedforward/feedback adaptive-delay algorithm with applications to piezo-based raster tracking," IEEE Trans. Control Systems Technology, vol. 20, no. 2, pp. 416-423, March 2012.

[19] S. Salapaka, A. Sebastian, J. P. Cleveland, and M. V. Salapaka, "High bandwidth nano-positioner: A robust control approach," Review of Scientific Instruments, vol. 73, no. 9, pp. 3232-3241, 2002.

[20] A. Bazaei, Y. K. Yong, and S. O. R. Moheimani, "Combining spiral scanning and internal model control for sequential AFM imaging at video rate," IEEE/ASME Transactions on Mechatronics, vol. 22, no. 1, pp. 371-380, Feb. 2017.

[21] J.-W. Wu, Y.-T. Lin, Y.-T. Lo, W.-C. Liu, and L.-C. Fu, "Lissajous hierarchical local scanning to increase the speed of atomic force microscopy," IEEE Transactions on Nanotechnology, vol. 14, no. 5, pp. 810-819, Sept. 2015.

[22] M. S. Rana, H. R. Pota, and I. R. Petersen, "Spiral scanning with improved control for faster imaging of AFM," IEEE Transactions on Nanotechnology, vol. 13, no. 3, pp. 541-550, May 2014.

[23] T. Tuma, J. Lygeros, V. Kartik, A. Sebastian, and A. Pantazi, "Highspeed multiresolution scanning probe microscopy based on Lissajous scan trajectories," Nanotechnology, vol. 23, no. 18, p. 185501, Apr. 2012.

[24] Y. K. Yong, S. O. R. Moheimani, and I. R. Petersen, "High-speed cycloid-scan atomic force microscopy," Nanotechnology, vol. 21, no. 36, p. 365503, Aug. 2010.

[25] Y. R. Teo, Y. Yong, and A. J. Fleming, "A comparison of scanning methods and the vertical control implications for scanning probe microscopy," Asian Journal of Control, vol. 28, no. 2, p. 65, Dec. 2016.

[26] B. Hartman and S. B. Andersson, "Feature tracking for high speed AFM imaging of biopolymers," International Journal of Molecular Sciences, vol. 19, no. 4, p. 1044, Mar. 2018.

[27] Y. Wen, J. Song, X. Fan, D. Hussain, H. Zhang, and H. Xie, "Fast specimen boundary tracking and local imaging with scanning probe microscopy," Scanning, vol. 2018, no. 4, pp. 1-11, Mar. 2018.

[28] K. Zhang, T. Hatano, T. Tien, G. Herrmann, C. Edwards, S. C. Burgess, and M. Miles, "An adaptive non-raster scanning method in atomic force microscopy for simple sample shapes," Measurement Science and Technology, vol. 26, no. 3, p. 035401 (12 pages), Feb. 2015.

[29] P. Huang and S. B. Andersson, "Note: Fast imaging of DNA in atomic force microscopy enabled by a local raster scan algorithm," Review of Scientific Instruments, vol. 85, no. 6, p. 066101 (3 pages), June 2014.

[30] B. Song, N. Xi, R. Yang, K. W. C. Lai, and C. Qu, "Video rate atomic force microscopy (AFM) imaging using compressive sensing," in Proc. IEEE Conference on Nanotechnology, 2011, pp. 1056-1059.

[31] S. B. Andersson and L. Y. Pao, "Non-raster sampling in atomic force microscopy: A compressed sensing approach," in Proc. American Control Conference, 2012, pp. 2485-2490. 
[32] A. Chen, A. L. Bertozzi, P. D. Ashby, P. Getreuer, and Y. Lou, "Enhancement and recovery in atomic force microscopy images," in Excursions in Harmonic Analysis, Volume 2. Springer, 2013, pp. 311332.

[33] Y. Luo and S. B. Andersson, "A comparison of reconstruction methods for undersampled atomic force microscopy images," Nanotechnology, vol. 26, no. 50, p. 505703, 2015.

[34] G. Han, B. Lin, and Y. Lin, "Reconstruction of atomic force microscopy image using compressed sensing," Micron, vol. 105, pp. 1-10, 2018.

[35] B. D. Maxwell and S. B. Andersson, "A compressed sensing measurement matrix for atomic force microscopy," in Proc. American Control Conference, 2014, pp. 1631-1636.

[36] Y. Luo and S. B. Andersson, "A fast image reconstruction algorithm for compressed sensing-based atomic force microscopy," in Proc. American Control Conference, 2015, pp. 3503-3508.

[37] R. A. Braker, Y. Luo, L. Y. Pao, and S. B. Andersson, "Hardware demonstration of atomic force microscopy imaging via compressive sensing and $\mu$-path scans," in Proc. American Control Conf., 2018, pp. $6037-6042$.

[38] G. Han and B. Lin, "Optimal sampling and reconstruction of undersampled atomic force microscope images using compressive sensing," Ultramicroscopy, vol. 189, pp. 85 - 94, 2018.

[39] T. L. Jensen, T. Arildsen, J. Ostergaard, and T. Larsen, "Reconstruction of undersampled atomic force microscopy images: Interpolation versus basis pursuit," in Proc. Int. Conf. Signal-Image Technology InternetBased Systems, 2013, pp. 130-135.

[40] C. S. Oxvig, T. Arildsen, and T. Larsen, "Structure assisted compressed sensing reconstruction of undersampled AFM images," Ultramicroscopy, vol. 172, no. Supplement C, pp. 1-9, 2017.

[41] A. Y. Carmi, "Compressive system identification," in Compressed Sensing \& Sparse Filtering. Springer, 2014, pp. 281-324.

[42] E. Candès and J. Romberg, "Sparsity and incoherence in compressive sampling," Inverse problems, vol. 23, no. 3, p. 969, 2007.

[43] S. Becker, J. Bobin, and E. Candès, "NESTA: A fast and accurate firstorder method for sparse recovery," SIAM Journal on Imaging Sciences, vol. 4, no. 1, pp. 1-39, 2011.

[44] E. J. Candès, Y. C. Eldar, D. Needell, and P. Randall, "Compressed sensing with coherent and redundant dictionaries," Applied and Computational Harmonic Analysis, vol. 31, no. 1, pp. 59 - 73, 2011.

[45] E. Meyer, "Atomic force microscopy," Progress in Surface Science, vol. 41, no. 1, pp. 3-49, 1992.

[46] K. Kuhnen, "Modeling, identification and compensation of complex hysteretic nonlinearities: A modified Prandtl-Ishlinskii approach," European J. Control, vol. 9, no. 4, pp. 407-418, 2003.

[47] R. A. Braker and L. Y. Pao, "A comparison of tracking step inputs with a piezo stage using MPC and saturated LQG control," IEEE Trans. Control System Tech., 2019, (under review). [Online]. Available: https://rabraker.com/publication_pdf/braker_pao_tcst_2019.pdf

[48] G. M. Clayton, S. Tien, K. K. Leang, Q. Zou, and S. Devasia, "A review of feedforward control approaches in nanopositioning for high-speed SPM," Journal of Dynamic Systems, Measurement, and Control, vol. 131, pp. 061 101-061 101-19, Oct. 2009.

[49] Z. Wang, A. C. Bovik, H. R. Sheikh, and E. P. Simoncelli, "Image quality assessment: from error visibility to structural similarity," IEEE Trans. Image Processing, vol. 13, no. 4, pp. 600-612, Apr. 2004.

[50] T. Ando, T. Uchihashi, and T. Fukuma, "High-speed atomic force microscopy for nano-visualization of dynamic biomolecular processes," Progress in Surface Science, vol. 83, no. 7, pp. 337 - 437, 2008. [Online]. Available: http://www.sciencedirect.com/science/article/ pii/S0079681608000464

[51] J. Stirling and R. Woolley, "Scanning probe image wizard - matlab toolbox," https://sourceforge.net/projects/spiw/, accessed: 2019-4-27.

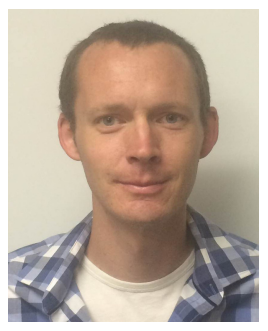

Roger A. Braker earned a B.A. degree in physics from the University of Oklahoma in 2005 and M.S. and $\mathrm{Ph} . \mathrm{D}$. degrees in electrical engineering from the University of Colorado in 2017 and 2019, respectively.

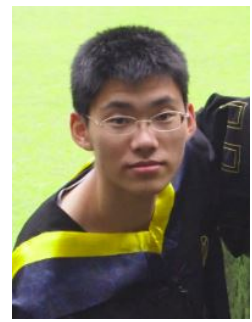

Yufan Luo received dual B.S. degrees in automation and in management science from Zhejiang University, Zhejiang, China, in 2011, an M.S. degree in industrial and operations engineering from the University of Michigan, Ann Arbor, MI, USA, in 2012, and a Ph.D. degree in systems engineering from Boston University in 2019. He is currently with Juniper Networks, Sunnyvale, CA.

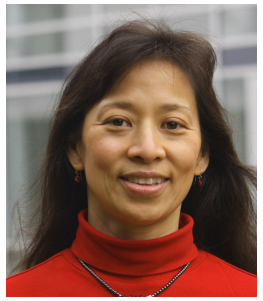

Lucy Y. Pao is the Palmer Endowed Chair Professor in the Electrical, Computer, and Energy Engineering Department at the University of Colorado Boulder. She earned B.S., M.S., and Ph.D. degrees in Electrical Engineering from Stanford University. Her research has primarily focused on engineering control systems, with applications ranging from atomic force microscopes to multi-megawatt wind energy systems. She is a Fellow of the IEEE and the International Federation of Automatic Control (IFAC). Selected recent awards include the 2012 IEEE Control Systems Magazine Outstanding Paper Award, the 2015 Society for Industrial and Applied Mathematics (SIAM) Journal on Control and Optimization Best Paper Prize, the 2017 Control Engineering Practice Award from the American Automatic Control Council, the Scientific Award 2017 from the European Academy of Wind Energy, and the 2019 Nyquist Lecturer Award from the ASME Dynamic Systems \& Control Division. Selected recent and current professional society activities include being a Fellow of the Renewable and Sustainable Energy Institute (2009-present), General Chair of the 2013 American Control Conference, member of the IEEE Control Systems Society (CSS) Board of Governors (2011-2013 and 2015), IEEE CSS Fellow Nominations Chair (2016-2018), member of the IFAC Fellow Selection Committee (2014-2017 and 2017-2020), and member of the IFAC Executive Board (2017-2020).

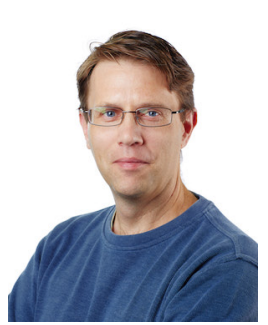

Sean B. Andersson received a B.S. degree in engineering and applied physics from Cornell University, Ithaca, NY, USA, in 1994, an M.S. degree in mechanical engineering from Stanford University, Stanford, CA, USA, in 1995, and a Ph.D. degree in electrical and computer engineering from the University of Maryland, College Park, MD, USA, in 2003. He has worked at AlliedSignal Aerospace and Aerovironment, Inc. and is currently an Associate Professor of mechanical engineering and of systems engineering with Boston University, Boston, MA, USA. His research interests include systems and control theory with applications in scanning probe microscopy, dynamics in molecular systems, and robotics. He received an NSF CAREER award in 2009, is a senior member of the IEEE, and was an associate editor for the IEEE Transactions on Automatic Control (2014-2018) and for the SIAM Journal on Control and Optimization (2013-2018). 